\title{
COCYCLES AND ALMOST PERIODICITY
}

\section{WOJCIECH CHOJNACKI}

\section{Dedicated to my wife Hania}

\begin{abstract}
We discuss different types of almost periodicity of trajectories of cocycles on compact solenoidal groups in relation to various notions of triviality of these cocycles.
\end{abstract}

\section{Introduction}

Let $G$ be a compact solenoidal group, that is, a compact Abelian group containing, as a dense subgroup, the image of $\mathbb{R}$ by a continuous homomorphism, say $\alpha$. We shall assume that $G$ is not a circle by requiring $\alpha$ to be injective. The dual group $\hat{G}$ of $G$ may then be identified with a dense subgroup of $\mathbb{R}$ : via the adjoint to $\alpha, \hat{G}$ embeds into $\mathbb{R} \simeq \mathbb{R}$ as a group which is not isomorphic to the group of integers and hence is dense in $\mathbb{R}$. For each $\mu \in \hat{G}$, where $\hat{G}$ is regarded as a subgroup of $\mathbb{R}, \chi_{\mu}$ will stand for the continuous character of $G$ such that $\chi_{\mu}(\alpha(x))=e^{i \mu x}$ for all $x \in \mathbb{R}$. If $\mu \in \mathbb{R} \backslash \hat{G}$, then $\chi_{\mu}$ denotes, by definition, the function identically zero on $G$.

Functions with values in the circle group $T$ are said to be unitary. A continuous cocycle on $G$ is a unitary continuous function $Y$ on $G \times \mathbb{R}$ satisfying, for all $s, t \in \mathbb{R}$ and all $g \in G$, the cocycle identity

$$
Y(g, s+t)=Y(g, s) Y(g+\alpha(s), t) .
$$

In the present paper cocycles other than continuous do not appear, so we abbreviate 'continuous cocycles' to 'cocycles'. A cocycle $Y$ on $G$ is trivial if there exist $\gamma \in \mathbb{R}$ and a unitary $\lambda$-measurable function $X$ on $G$ such that, given $t \in \mathbb{R}$,

$$
Y(g, t)=e^{i \gamma t} X(g) \overline{X(g+\alpha(t))}
$$

for $\gamma$-almost all $g$ in $G$, where $\lambda$ is the Haar measure on $G$. Any pair $(\gamma, X)$ satisfying (*) will be referred to as a standard representation of $Y$. A cocycle $Y$ on $G$ will be called $c$-trivial if it has a standard representation $(\gamma, X)$ in which $X$ is a continuous function on $G$. Now $c$-trivial cocycles on $G$ can be constructed in an obvious manner. Modifying slightly an argument contained in [3], one can easily prove that if $\left(a_{n}\right)_{n \in \mathbb{N}}$ is a sequence in $\hat{G}$ such that $n^{-\frac{2}{3}} \leqslant a_{n} \leqslant 2 n^{-\frac{2}{3}}$ for all $n \in \mathbb{N}$, then setting

$$
Y(g, t)=\prod_{n-1}^{\infty} \exp \left\{i a_{n}\left[\left(1-e^{i a_{n} t}\right) \chi_{a_{n}}(g)+\left(1-e^{-i a_{n} t}\right) \chi_{-a_{n}}(g)\right]\right\} \quad(g \in G, t \in \mathbb{R})
$$

defines a trivial non-c-trivial cocycle on $G$. H. Helson and J.-P. Kahane [8] gave a construction of a non-trivial cocycle on $G$.

Received 23 May 1985; revised 3 March 1986.

1980 Mathematics Subject Classification 43A17. 
Given a cocycle $Y$ on $G$, each function of the form

$$
t \longrightarrow Y(g, t) \quad(g \in G)
$$

will be referred to as a trajectory of $Y$.

In this paper we exhibit various relations between different types of almost periodicity of trajectories of cocycles and different notions of triviality of cocycles. In Section 1 we prove that $c$-trivial cocycles are the only trivial cocycles all of whose trajectories are Hartman almost periodic. Next, generalizing results of D. Lowdenslager and C. G. R. Carlson, we characterize trivial cocycles as those which have all trajectories almost periodic in a non-classical generalized sense; half of this result appears in Section 2, the other half in Section 3. Section 3 presents, inter alia, a theorem to the effect that the trajectories of a cocycle are Ryll-Nardzewski almost periodic if and only if the cocycle is either $c$-trivial or non-trivial.

\section{Besicovitch and Hartman almost periodicity of trajectories of trivial cocycles}

Given $\mu \in \mathbb{R}$ and a complex locally integrable function $f$ on $\mathbb{R}$, the limit

$$
\lim _{T \rightarrow \infty} \frac{1}{2 T} \int_{-T}^{T} f(t) e^{-i \mu t} d t
$$

if it exists, is called the $\mu$ th Fourier coefficient of $f$ and is denoted by $\mathscr{F} f(\mu)$. A complex locally integrable function is said to be Hartman almost periodic (or, more briefly, $\mathrm{H}$ a.p.) if all of its Fourier coefficients exist (cf. [9]). For an $\mathrm{H}$ a.p. function $f$, the set $\{\mu \in \mathbb{R}: \mathscr{F} f(\mu) \neq 0\}$ is called the spectrum of $f$.

A complex measurable function on $\mathbb{R}$ is said to be Besicovitch $\mathbf{B}^{2}$ almost periodic (or, more briefly, $\mathbf{B}^{2}$ a.p.) if it is the limit of a sequence of trigonometric polynomials in the pseudonorm

$$
\|u\|_{\mathrm{B}^{2}}=\lim _{T \rightarrow \infty}\left(\frac{1}{2 T} \int_{-T}^{T}|u(t)|^{2} d t\right)^{\frac{1}{2}} .
$$

Any $\mathrm{B}^{2}$ a.p. function $f$ is $\mathrm{H}$ a.p., its spectrum is at most countable, and

$$
\|f\|_{\mathrm{B}^{2}}=\sum_{n=1}^{\infty}\left|\mathscr{F} f\left(\mu_{n}\right)\right|^{2},
$$

where $\left\{\mu_{n}: n \in \mathbb{N}\right\}$ is the spectrum of $f$. Conversely, if a complex measurable function $f$ on $\mathbb{R}$ satisfies $\|f\|_{\mathrm{B}^{2}}<+\infty$ and there exist $\mu_{n} \in \mathbb{R}(n \in \mathbb{N})$ such that the $\mathscr{F} f\left(\mu_{n}\right)$ exist and the above identity holds, then $f$ is $\mathrm{B}^{2}$ a.p. and $\left\{\mu_{n}: n \in \mathbb{N}\right\}$ contains the spectrum of $f$.

We owe the following result to Lowdenslager (cf. [7, p. 20]).

THEOREM 1.1. Almost all trajectories of a trivial cocycle on $G$ are $\mathrm{B}^{2}$ a.p.

The natural question arises whether the above statement is valid when 'almost all' is replaced by 'all'. For $c$-trivial cocycles the answer is clearly yes-all trajectories of such cocycles are uniformly almost periodic. In the general case, however, the answer is no. The main result of this section will be the assertion that the property of having all trajectories $\mathrm{H}$ a.p. characterizes $c$-trivial cocycles among trivial cocycles.

For the convenience of the reader and for some technical reasons we begin by re-proving Lowdenslager's theorem.

For $1 \leqslant p \leqslant+\infty$, let $L^{p}(G)$ be the $p$ th Lebesgue space based on $\lambda$ and let $\|\cdot\|_{p}$ 
be the corresponding norm. Given a function $F$ in $L^{1}(G)$ and $\mu \in \mathbb{R}$, let $\mathscr{F} F(\mu)$ denote the $\mu$ th Fourier coefficient of $F$, that is,

$$
\mathscr{F} F(\mu)=\int_{G} F(g) \overline{\chi_{\mu}(g)} d \lambda(g) .
$$

Let $Y$ be a trivial cocycle on $G$ with a standard representation $(\gamma, X)$. The action $(g, t) \rightarrow g+\alpha(t)$ of $\mathbb{R}$ on $G$ is ergodic, so by Birkhoff's ergodic theorem, for any $\mu \in \mathbb{R}$, the limit

$$
\lim _{T \rightarrow \infty} \frac{1}{2 T} \int_{-T}^{T} \overline{X(g+\alpha(t))} \chi_{\gamma-\mu}(g+\alpha(t)) d t
$$

exists for $\lambda$-almost all $g$ in $G$ and is equal to $\overline{\mathscr{F} X(\gamma-\mu)}$. Therefore

$$
\mathscr{F} Y(g, \cdot)(\mu)=\overline{\mathscr{F} X(\gamma-\mu)} X(g) \chi_{\mu-\gamma}(g)
$$

except on a $\lambda$-null set $E_{\mu}$. Let $S=\{\mu \in \mathbb{R}: \mathscr{F} X(\gamma-\mu) \neq 0\}$. Since the set $S$ is countable, the set $E=\bigcup_{\mu \in S} E_{\mu}$ is $\lambda$-null. Since $X$ is a unitary function, it follows from (1.1) and Plancherel's theorem that for $\lambda$-almost all $g$ in $G \backslash E$,

$$
\sum_{\mu \in S}|\mathscr{F} Y(g, \cdot)(\mu)|^{2}=\sum_{\mu \in S}|\mathscr{F} X(\gamma-\mu)|^{2}=\|X\|_{2}^{2}=1 .
$$

Hence we reach the conclusion of Lowdenslager's theorem on utilizing the criterion of $\mathrm{B}^{2}$ almost periodicity mentioned above and the fact that the $\mathrm{B}^{2}$ norm of each trajectory of $Y$ is equal to 1 .

We state now the main result of this section.

THEOREM 1.2. A trivial cocycle $Y$ on $G$ is c-trivial if and only if all trajectories of $Y$ are $\mathrm{H}$ a.p.

The proof of the necessity is clear. The proof of the sufficiency exploits an argument essentially due to H. Furstenberg (cf. [6]). It will be convenient to give this proof by proceeding in two steps.

Proposition 1.3. Let $Y$ be a cocycle on $G$. Let $\gamma$ be a real number and $Z$ a complex function on $G$ having a point of continuity, such that for each $t \in \mathbb{R}$ and each $g \in G$,

Then $Z$ is continuous.

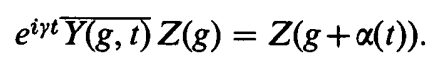

Proof. Given $g$ in $G$, let $\Omega(g)$ denote the oscillation of $Z$ at $g$, that is, $\inf \{\operatorname{diam} Z(U): U$ an open neighbourhood of $g$ \}. It is clear that the function $g \rightarrow \Omega(g)$ is upper semicontinuous.

For any $\delta>0$, let $F_{\delta}=\{g \in G: \Omega(g) \geqslant \delta\}$. Each $F_{\delta}$ is closed and, in view of (1.2), invariant under the $\mathbb{R}$ action on $G$, so each is either empty or all of $G$. From this the proposition follows immediately.

We complete now the proof of Theorem 1.2 by reasoning as follows. Suppose that $Y$ is a trivial cocycle all of whose trajectories are $\mathrm{H}$ a.p. Let $(\gamma, X)$ be a standard representation of $Y$. Choose $\mu$ in the coset $\hat{G}+\gamma$ so that $\mathscr{F} X(\gamma-\mu) \neq 0$. Since all the functions

$$
g \longrightarrow \frac{1}{2 T} \int_{-T}^{T} Y(g, t) e^{-i \mu t} d t \quad(T>0)
$$


are continuous, the function $g \rightarrow \mathscr{F} Y(g, \cdot)(\mu)$ is of the first Baire class and so, by Baire's theorem (cf. [10, Theorem 27.10.1]), has a point of continuity. Clearly, the function $Z$ defined by

$$
Z(g)=\mathscr{F} Y(g, \cdot)(\mu) \chi_{\gamma-\mu}(g) \quad(g \in G)
$$

also has a point of continuity. By the cocycle relation, for each $t \in \mathbb{R}$ and each $g \in G$,

$$
\mathscr{F} Y(g+\alpha(t), \cdot)(\mu) Y(g, t)=e^{i \mu t} \mathscr{F} Y(g, \cdot)(\mu),
$$

so condition (1.2) is fulfilled. By Proposition 1.3, $Z$ is continuous, whence, on account of (1.1), $X$ can be taken to be continuous.

The proof is complete.

\section{Generalized almost periodicity of trajectories of trivial cocycles}

Theorem 1.2 shows that a trajectory of a trivial cocycle may fail to be almost periodic in any classical sense. In this section, we prove that every trajectory of a trivial cocycle is almost periodic in a generalized sense. Actually we demonstrate that each trajectory of a trivial non-c-trivial cocycle is almost periodic in at least two generalized senses as introduced in this section, while for trajectories of $c$-trivial cocycles all types of generalized almost periodicity coincide with uniform almost periodicity.

We start with a technical result.

Proposition 2.1. Let $Y_{1}, \ldots, Y_{n}$ be cocycles on $G$, and let $a_{1}, \ldots, a_{n}$ be complex and $t_{1}, \ldots, t_{n}$ real numbers. Then the function

$$
g \longrightarrow \sup \left\{\left|\sum_{i=1}^{n} a_{i} Y_{i}\left(g, t+t_{i}\right)\right|: t \in \mathbb{R}\right\}
$$

is continuous.

Proof. Let $\left(\Sigma_{k}\right)_{k \in \mathbb{N}}$ be an increasing sequence of finite subsets of $\mathbb{R}$ such that $\bigcup_{k-1}^{\infty} \Sigma_{k}$ is dense in $\mathbb{R}$. Given $g \in G$ and $\alpha=\left(\alpha_{1}, \ldots, \alpha_{n}\right) \in \mathbb{T}^{n}$, put

$$
\begin{aligned}
f_{k}(g, \alpha) & =\max \left\{\left|\sum_{i=1}^{n} a_{i} \alpha_{i} Y_{i}\left(g, t+t_{i}\right)\right|: t \in \Sigma_{k}\right\} \quad(k \in \mathbb{N}), \\
f(g, \alpha) & =\sup \left\{\left|\sum_{i=1}^{n} a_{i} \alpha_{i} Y_{i}\left(g, t+t_{i}\right)\right|: t \in \mathbb{R}\right\} .
\end{aligned}
$$

For each $g \in G$, the functions $\alpha \rightarrow f_{k}(g, \alpha)$ are equicontinuous and tend pointwise to the continuous function $\alpha \rightarrow f(g, \alpha)$ as $k \rightarrow \infty$; hence, by the compactness of $\mathbb{T}^{n}$, the functions $\alpha \rightarrow f_{k}(g, \alpha)$ tend to the function $\alpha \rightarrow f(g, \alpha)$ uniformly. Each function $G \ni g \rightarrow f_{k}(g, \cdot) \in C\left(\mathbb{T}^{n}\right)$ (where $C\left(\mathbb{T}^{n}\right)$ is the Banach space of all complex continuous functions on $\mathbb{T}^{n}$ ) is continuous, so, by Baire's theorem, the function $G \ni g \rightarrow f(g, \cdot) \in C\left(\mathbb{T}^{n}\right)$ has a point of continuity. The proposition will follow once we show that this function is actually continuous.

Given $g \in G, \alpha \in \mathbb{T}^{n}$, and $t \in \mathbb{R}$, the cocycle relation implies that

$$
f(g+\alpha(t), \alpha)=f\left(g, \alpha_{g, t}\right),
$$


where $\alpha_{g, t}$ denotes the element $\left(\alpha_{1} \overline{Y_{1}(g, t)}, \ldots, \alpha_{n} \overline{Y_{n}(g, t)}\right)$ of $\mathbb{T}^{n}$. Thus, for each $g, g^{\prime} \in G$, each $\alpha \in \mathbb{T}^{n}$, and each $t \in \mathbb{R}$,

$$
\begin{aligned}
\left|f(g+\alpha(t), \alpha)-f\left(g^{\prime}+\alpha(t), \alpha\right)\right| \leqslant & \left|f\left(g, \alpha_{g, t}\right)-f\left(g^{\prime}, \alpha_{g, t}\right)\right|+\left|f\left(g^{\prime}, \alpha_{g, t}\right)-f\left(g^{\prime} \alpha_{g^{\prime}, t}\right)\right| \\
\leqslant & \left|f\left(g, \alpha_{g, t}\right)-f\left(g^{\prime}, \alpha_{g, t}\right)\right| \\
& +\max \left\{\left|Y_{i}(g, t)-Y_{i}\left(g^{\prime}, t\right)\right|: 1 \leqslant i \leqslant n\right\} \cdot \sum_{i=1}^{n}\left|a_{i}\right| .
\end{aligned}
$$

Consequently, the oscillation function $\Omega$ for $g \rightarrow f(g, \cdot)$ satisfies

$$
\Omega(g+\alpha(t)) \leqslant \Omega(g)
$$

for each $g \in G$ and each $t \in \mathbb{R}$. Substituting $g$ for $g+\alpha(t)$ and $\alpha(-t)$ for $\alpha(t)$ in this inequality leads to the reverse inequality. We thus see that $\Omega$ is invariant under the $\mathbb{R}$ action on $G$. Now we complete the proof by repeating the argument used in the proof of Proposition 1.3.

A linear continuous functional $m$ on $L^{\infty}(\mathbb{R})$ is called a Banach mean on $L^{\infty}(\mathbb{R})$ if it satisfies the following conditions:

(i) $m(1)=1=\|m\|$,

(ii) $m\left(T_{s} f\right)=m(f)$ for each $f \in L^{\infty}(\mathbb{R})$ and each $s \in \mathbb{R}$, where $T_{s} f$ denotes the translate of $f$ by $s$.

As is known, there exist at least $2^{\mathfrak{C}}$ different Banach means on $L^{\infty}(\mathbb{R})$ (cf. [12]).

If $m$ is a Banach mean on $L^{\infty}(\mathbb{R})$, then under the scalar product derived from the map $(f, g) \rightarrow m(f \bar{g})\left(f, g \in L^{\infty}(\mathbb{R})\right)$, the quotient space

$$
H_{m}=L^{\infty}(\mathbb{R}) /\left\{f \in L^{\infty}(\mathbb{R}): m\left(|f|^{2}\right)=0\right\}
$$

is a pre-Hilbert space. The functions $x \rightarrow e^{i \mu x}(\mu \in \mathbb{R})$ generate an orthonormal set in $H_{m}$. Given $f \in L^{\infty}(\mathbb{R})$ and $\mu \in \mathbb{R}$, the $\mu$ th Fourier coefficient $\mathscr{F}_{m} f(\mu)$ of $f$ with respect to $m$ is defined as the Fourier coefficient of the class in $H_{m}$ of $f$ with respect to the class in $H_{m}$ of $x \rightarrow e^{i \mu x}$. Of course, $\mathscr{F}_{m} f(\mu)=m_{x}\left(f(x) e^{-i \mu x}\right)$, where the subscript $x$ indicates that the action of $m$ refers to the variable $x$. By Bessel's inequality, one has

$$
\sum_{\mu \in \mathbf{R}}\left|\mathscr{F}_{m} f(\mu)\right|^{2} \leqslant m\left(|f|^{2}\right)
$$

In the case of equality in the latter relation, $f$ is said to be $\mathrm{B}_{m}^{2}$ almost periodic (or, more briefly, $B_{m}^{2}$ a.p.). It is clear that a function in $L^{\infty}(\mathbb{R})$ is $B_{m}^{2}$ a.p. if and only if its class in $H_{m}$ is the limit of a sequence of the classes in $H_{m}$ of trigonometric polynomials on $\mathbb{R}$.

A linear continuous functional $\xi$ on $L^{\infty}(G)$ is said to be localized at a point $g$ in $G$ if, given an open neighbourhood $U$ of $g$ and $F, H \in L^{\infty}(G)$, the equality $G(h)=H(h)$ for $\lambda$-almost all $h$ in $U$ implies that $\xi(F)=\xi(H)$. As is known, for each $g$ in $G$, there is a linear multiplicative (continuous) functional on $L^{\infty}(G)$ localized at $g$ (cf. [13, Proposition 15.4.2]).

We are now in a position to state the following theorem. 
THEOREM 2.2. Let $Y$ be a trivial cocycle on $G$ with a standard representation $(\gamma, X)$. Let $g$ be an element of $G$ and $\phi$ a linear multiplicative functional on $L^{\infty}(G)$ localized at $g$. Then there exists a Banach mean $m$ on $L^{\infty}(\mathbb{R})$ such that for all $\mu \in \mathbb{R}$,

$$
\mathscr{F}_{m} Y(g, \cdot)(\mu)=\overline{\mathscr{F} X(\gamma-\mu)} \phi(X) \chi_{\mu-\gamma}(g) .
$$

Proof. Let $\Psi$ be the smallest translation-invariant subspace of $L^{\infty}(\mathbb{R})$ containing all the functions $t \rightarrow Y(g, t+s) e^{i \mu t}(s, \mu \in \mathbb{R})$ and the function identically equal to 1 . Given $a_{0}, \ldots, a_{n} \in \mathbb{C}$ and $t_{1}, \ldots, t_{n}, \mu_{1}, \ldots, \mu_{n} \in \mathbb{R}$, let $\psi$ be the function on $G \times \mathbb{R}$ defined by

$$
\psi(h, t)=a_{0}+\sum_{i=1}^{n} a_{i} Y\left(h, t+t_{i}\right) e^{-i \mu_{i} t} \quad(h \in G, t \in \mathbb{R}) .
$$

By (1.1), for $\lambda$-almost all $h$ in $G$,

$$
\mathscr{F} \psi(h, \cdot)(0)=a_{0}+\sum_{i=1}^{n} a_{i} e^{i \mu_{i} t_{i}} \overline{\mathscr{F} X\left(\gamma-\mu_{i}\right)} X(h) \chi_{\mu_{i}-\gamma}(h) .
$$

Hence, for each open neighbourhood $U$ of $g$,

$$
\text { ess sup }\left\{\left|a_{0}+\sum_{i=1}^{n} a_{i} e^{i \mu_{i} t_{i}} \overline{\mathscr{F} X\left(\gamma-\mu_{i}\right)} X(h) \chi_{\mu_{i}-\gamma}(h)\right|: h \in U\right\} \leqslant \sup \{|\psi(h, t)|: h \in U, t \in \mathbb{R}\} .
$$

Since, by Proposition 2.1, the function $h \rightarrow \sup \{|\psi(h, t)|: t \in \mathbb{R}\}$ is continuous and since $\phi$ is localized at $g$, it follows that

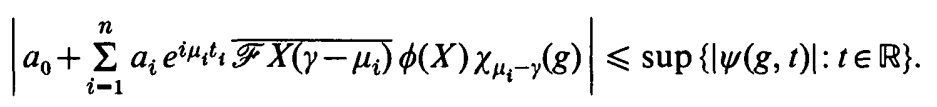

Thus by assigning to each function of the form $t \rightarrow \psi(g, t)$ the corresponding number

$$
a_{0}+\sum_{i=1}^{n} a_{i} e^{i \mu_{i} t_{i}} \overline{\mathscr{F} X\left(\gamma-\mu_{i}\right)} \phi(X) \chi_{\mu_{i}-\gamma}(g),
$$

we define a linear continuous functional $m$ on $\Psi$ such that $m(1)=1=\|m\|$. One verifies at once that $m$ is translation-invariant, that is, $m\left(T_{s} f\right)=m(f)$ for all $f \in \Psi$ and all $s \in \mathbb{R}$. We also see that (2.1) is satisfied. To complete the proof, it suffices to extend $n$ to a Banach mean on $L^{\infty}(\mathbb{R})$, which is possible by virtue of an invariant prolongation theorem due to Agnew and Morse [1; 5, Theorem 3.3.1].

We now state the main conclusion of this section.

THEOREM 2.3. Every trajectory of a trivial cocycle on $G$ is $\mathbf{B}_{m}^{2}$ a.p. for some Banach mean $m$ on $L^{\infty}(\mathbb{R})$.

Proof. Let $Y$ be a trivial cocycle on $G$ with a standard representation $(\gamma, X)$. Let $g$ be an element of $G$, and $\phi$ a linear multiplicative functional on $L^{\infty}(G)$ localized at $g$. By Theorem 2.2, there is a Banach mean $m$ on $L^{\infty}(\mathbb{R})$ such that the Fourier coefficients of $t \rightarrow Y(g, t)$ with respect to $m$ satisfy (2.1). As $|\phi(X)|=\phi(|X|)=1$, we have

$$
\sum_{\mu \in \mathbb{R}}\left|\mathscr{F}_{m} Y(g, \cdot)(\mu)\right|^{2}=\sum_{\mu \in \mathbb{R}}|\mathscr{F} X(\gamma-\mu)|^{2}=1 .
$$

But this means that the trajectory $t \rightarrow Y(g, t)$ is $\mathrm{B}_{m}^{2}$ a.p.

The proof is complete. 
The stronger version of Theorem 2.3 mentioned at the beginning of this section can be deduced from the following result.

THEOREM 2.4. Let $Y$ be a trivial cocycle on $G$. If there exist $g \in G$ and $\mu \in \mathbb{P}$ such that $\mathscr{F}_{m} Y(g, \cdot)(\mu)$ is one and the same non-zero number for all the Banach means $m$ on $L^{\infty}(\mathbb{R})$ for which the trajectory $t \rightarrow Y(g, t)$ is $\mathrm{B}_{m}^{2}$ a.p., then the cocycle $Y$ is c-trivial. Conversely, if $Y$ is c-trivial, then for each $g \in G$, each $\mu \in \mathbb{R}$, and each Banach mean $m$ on $L^{\infty}(\mathbb{R})$,

$$
\mathscr{F}_{m} Y(g, \cdot)(\mu)=\mathscr{F} Y(g, \cdot)(\mu) .
$$

Proof. To prove the first assertion, let $Y$ be a trivial cocycle on $G$ with a standard representation $(\gamma, X)$, let $g$ be an element of $G$, and let $\mu$ be a real number such that $\mathscr{F}_{m} Y(g, \cdot)(\mu)$ is one and the same non-zero number for all the Banach means $m$ on $L^{\infty}(\mathbb{R})$ for which the trajectory $t \rightarrow Y(g, t)$ is $\mathrm{B}_{m}^{2}$ a.p. Let $\phi$ be a linear multiplicative functional on $L^{\infty}(G)$ localized at $g$. Given $h \in G$, denote by $\phi_{h}$ the linear multiplicative functional on $L^{\infty}(G)$ defined by

$$
\phi_{h}(F)=\phi\left(T_{h-g} F\right) \quad\left(F \in L^{\infty}(G)\right) .
$$

For each $h \in G$, put $Z(h)=\phi_{h}(X)$. We claim that $Z$ is continuous at $g$.

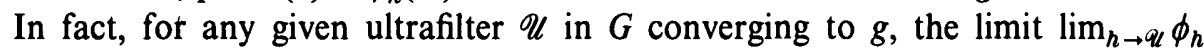
taken in the *-weak topology on $L^{\infty}(G)$ is a linear multiplicative functional on $L^{\infty}(G)$ localized at $g$. In view of the hypothesis, Theorem 2.2 implies that the value of this functional at $X$ is equal to $\phi(X)$. Hence $\lim _{h \rightarrow \mathscr{U}} Z(h)=Z(g)$, as claimed.

For any $h \in G, \phi_{h}$ acts on $C(G)$ as the evaluation functional at $h$. Thus, for each $h \in G$ and each $t \in \mathbb{R}$,

$$
Y(h, t)=\phi_{h}(Y(\cdot, t))=e^{i \gamma t} Z(h) \overline{Z(h+\alpha(t))} .
$$

Moreover, $|Z(h)|=\phi_{h}(|Z|)=1$, and so (1.2) holds. By Proposition 1.3, $Z$ is continuous. The first part of the theorem is thus established.

The second part is an immediate consequence of the well-known fact that

$$
f \longrightarrow \lim _{T \rightarrow \infty} \frac{1}{2 T} \int_{-T}^{T} f(t) d t
$$

is the only invariant mean on the space of all uniformly almost periodic functions on $\mathbb{R}$.

\section{Generalized Fourier transforms and Ryll-Nardzewski almost perodicity of trajectories of cocycles}

In this section we study in detail the ranges of the Fourier transforms, with respect to Banach means, of trajectories of cocycles. This will be the basis for proving the converse to Theorem 2.3 and for discussing Ryll-Nardzewski almost periodicity of trajectories of cocycles.

THEOREM 3.1. Let $Y$ be a cocycle on $G$, let $g$ be an element of $G$, and let $m$ be a Banach mean on $L^{\infty}(\mathbb{R})$. If $Y$ is trivial and $(\gamma, X)$ is its standard representation, then there exists a constant $c=c(Y, g, m)$ of modulus at most 1 such that for all $\mu \in \mathbb{R}$,

$$
\mathscr{F}_{m} Y(g, \cdot)(\mu)=c \overline{\mathscr{F} X(\gamma-\mu)} \chi_{\mu-\gamma}(g) .
$$

If $Y$ is not trivial, then for each $\mu \in \mathbb{R}$,

$$
\mathscr{F}_{m} Y(g, \cdot)(\mu)=0 .
$$


Proof. Given $F \in L^{2}(G)$ and $t \in \mathbb{R}$, set

$$
\left(U_{t} F\right)(g)=\overline{Y(g, t)} F(g+\alpha(t)) \quad(g \in G) .
$$

Each $U_{t}: F \rightarrow U_{t} F$ is a unitary operator in $L^{2}(G)$, and $U: t \rightarrow U_{t}$ is a strongly continuous one-parameter group in $L^{2}(G)$, which, in the sequel, will be referred to as the group associated with $Y$. Let $i \mathscr{A}$ be the infinitesimal generator of $U$. As is known, the self-adjoint operator $\mathscr{A}$ has simple pure point spectrum if $Y$ is trivial and purely continuous spectrum otherwise (cf. [7]).

Let $b \mathbb{R}$ be the Bohr compactification of $\mathbb{R}$, let $\beta$ be the canonical monomorphism from $\mathbb{R}$ into $b \mathbb{R}$, and $\eta$ the homomorphism from $b \mathbb{R}$ onto $G$ such that $\alpha=\eta \circ \beta$. Define a cocycle $Z$ on $b \mathbb{R}$ by setting

$$
Z(\omega, t)=Y(\eta(\omega), t) \quad(\omega \in \mathrm{b} \mathbb{R}, t \in \mathbb{R}) .
$$

Let $V$ be the group associated with $Z$ and let $i \mathscr{B}$ be its generator. Since, for each $t \in \mathbb{R}$, $\left(U_{t} 1,1\right)=\left(V_{t} 1,1\right)$ (the scalar products here being that of $L^{2}(G)$ and $L^{2}(\mathrm{~b} \mathbb{R})$, respectively), the spectra of $\mathscr{A}$ and $\mathscr{B}$ are of the same type.

It is easily seen that $C(\mathrm{bR})$ is an invariant subspace of $V$, and the restriction of $V$ to $C(\mathrm{~b} \mathbb{P})$ is, under the supremum norm on $C(\mathrm{~b} \mathbb{R})$, a strongly continuous one-parameter group of contractions. Denote by $\mathscr{C}$ the infinitesimal generator of this group and by $D(\mathscr{C})$ its domain.

For each $\mu \in \mathbb{R}$, write $\zeta_{\mu}$ for the continuous character of $b \mathbb{R}$ such that $\zeta_{\mu}(\beta(x))=e^{i \mu x}$ for all $x \in \mathbb{R}$.

Let $m$ be a Banach mean on $L^{\infty}(\mathbb{R})$.

It is readily verified that if $\omega$ is an element of $b \mathbb{R}$ and $P$ is a trigonometric polynomial on $b \mathbb{R}$, then

$$
m_{t}\left(\left(V_{t} P\right)(\omega)\right)=\sum_{\mu \in \mathbb{R}} \overline{\mathscr{F}_{m} Z(\omega, \cdot)(\mu)} \mathscr{F} P(\mu) \zeta_{\mu}(\omega)
$$

Hence, by approximation, for each $F \in D(\mathscr{C})$,

$$
m_{t}\left(\left(V_{t} \mathscr{C} F\right)(\omega)\right)=\sum_{\mu \in \mathbb{R}} \overline{\mathscr{F}_{m} Z(\omega, \cdot)(\mu)} \mathscr{F} \mathscr{C} F(\mu) \zeta_{\mu}(\omega)
$$

Since, for $h \in \mathbb{R} \backslash\{0\}$,

and since the functions

$$
m_{t}\left(\frac{\left(V_{t+h} F\right)(\omega)-\left(V_{t} F\right)(\omega)}{h}\right)=0
$$

$$
t \longrightarrow \frac{\left(V_{t+h} F\right)(\omega)-\left(V_{t} F\right)(\omega)}{h}
$$

tend uniformly to the function $t \rightarrow V_{t} \mathscr{C} F$ as $h \rightarrow 0$, it follows that $m_{t}\left(\left(V_{t} \mathscr{C} F\right)(\omega)\right)=0$. This together with (3.1) yields

$$
\sum_{\mu \in \mathbb{R}} \overline{\mathscr{F}_{m} Z(\omega, \cdot)(\mu)} \mathscr{F} \mathscr{C} F(\mu) \zeta_{\mu}(\omega)=0
$$

Since $D(\mathscr{C})$ is dense in $C(\mathrm{~b} \mathbb{R})$, it is also dense in $L^{2}(\mathrm{~b} \mathbb{R})$. Moreover, $D(\mathscr{C})$ is $V$-invariant and contained in $D(\mathscr{B})$, the domain of $\mathscr{B}$. Under these circumstances, a theorem of de Leeuw [4] implies that for any given $H$ in $D(\mathscr{B})$ there is an element $F$ of $D(\mathscr{C})$ such that $\|\mathscr{B} H-\mathscr{C} F\|_{2}$ is as small as we please; thus, in view of (3.2), we have

$$
\sum_{\mu \in \mathbb{R}} \overline{\mathscr{F}_{m} Z(\omega, \cdot)(\mu)} \mathscr{F} \mathscr{B} H(\mu) \zeta_{\mu}(\omega)=0
$$


The last relation shows that the element $\left(\mathscr{F}_{m} Z(\omega, \cdot)(\mu) \zeta_{-\mu}(\omega)\right)_{\mu \in \mathbf{R}}$ of $l^{2}(\mathbb{R})$ is orthogonal to the range of the operator carrying any $g$ in $D(\mathscr{B})$ to $(\mathscr{F} \mathscr{B} H(\mu))_{\mu \in \mathbf{R}}$. Since $\mathscr{B}$ is self-adjoint, this element must lie in $\mathscr{F}($ ker $\mathscr{B})$, the image of the kernel of $\mathscr{B}$ by the Fourier transform.

At this stage, it is easy to complete the proof of the second assertion. When the cocycle $Y$ is not trivial, then the operator $\mathscr{B}$ has purely continuous spectrum. In particular, $\operatorname{ker} \mathscr{B}=\{0\}$. Consequently, given $g$ in $G$, if we choose $\omega \in \mathrm{b} \mathbb{R}$ so that $g=\eta(\omega)$, then, for each $\mu \in \mathbb{R}$, we have

$$
\mathscr{F}_{m} Y(g, \cdot)(\mu)=\mathscr{F}_{m} Z(\omega, \cdot)(\mu)=0 .
$$

Passing to the proof of the first assertion, suppose that the cocycle $Y$ is trivial and $(\gamma, X)$ is its standard representation. Then the operator $\mathscr{B}$ has simple pure point spectrum and, in particular, the kernel of $\mathscr{B}$ is at most one-dimensional. By Theorem 2.2, for each $g \in G$, there is a Banach mean $n$ on $L^{\infty}(\mathbb{R})$ and $d$ in $\mathbb{T}$ such that for each $\mu \in \mathbb{R}$,

$$
\mathscr{F}_{n} Y(g, \cdot)(\mu)=d \overline{\mathscr{F} X(\gamma-\mu)} \chi_{\mu-\gamma}(g) \text {. }
$$

Choose $\omega$ in bR such that $g=\eta(\omega)$. We claim that for each $\mu \in \mathbb{R}$,

$$
\mathscr{F}_{n} Z(\omega, \cdot)(\mu) \zeta_{-\mu}(\omega)=d \overline{\mathscr{F} X(\gamma-\mu)} \zeta_{-\gamma}(\omega) .
$$

This is clear for $\mu$ ranging over the coset $\hat{G}+\gamma$. In particular, we have

But

$$
\sum_{\mu \in \hat{G}+\lambda}\left|\mathscr{F}_{n} Z(\omega, \cdot)(\mu)\right|^{2}=\sum_{\mu \in \hat{G}+\lambda}|\mathscr{F} X(\gamma-\mu)|^{2}=1 .
$$

$$
\sum_{\mu \in \mathbb{R}}\left|\mathscr{F}_{n} Z(\omega, \cdot)(\mu)\right|^{2} \leqslant 1,
$$

and so the left-hand side of (3.3) vanishes for $\mu \in \mathbb{R} \backslash(\hat{G}+\lambda)$. That the right-hand side of (3.3) vanishes for $\mu \in \mathbb{R} \backslash(\hat{G}+\lambda)$ is obvious.

By the previous analysis, (3.3) implies that $\left(\overline{\mathscr{F} X(\gamma-\mu)} \zeta_{-\gamma}(\omega)\right)_{\mu \in \mathbf{R}}$ is in $\mathscr{F}($ ker $\mathscr{B})$. Of course, $\left(\overline{\mathscr{F} X}(\gamma-\mu) \zeta_{-\gamma}(\omega)\right)_{\mu \in \mathbf{R}}$ actually spans $\mathscr{F}(\operatorname{ker} \mathscr{B})$. Thus if $m$ is any Banach mean on $L^{\infty}(\mathbb{R})$, then there is a constant $c$ such that for all $\mu \in \mathbb{R}$,

$$
\mathscr{F}_{m} Z(\omega, \cdot)(\mu) \zeta_{-\mu}(\omega)=c \overline{\mathscr{F} X(\gamma-\mu)} \zeta_{-\gamma}(\omega),
$$

whence

Noting that

$$
\mathscr{F}_{m} Y(g, \cdot)(\mu)=\overline{\mathscr{F} X(\gamma-\mu)} \chi_{\mu-\gamma}(g)
$$

$$
|c|^{2}=|c|^{2} \sum_{\mu \in \mathbf{R}}|\mathscr{F} X(\gamma-\mu)|^{2}=\sum_{\mu \in \mathbf{R}}\left|\mathscr{F}_{m} Y(g, \cdot)(\mu)\right|^{2} \leqslant 1
$$

completes the proof of the second assertion.

As an immediate consequence, we obtain the converse to Theorem 2.3.

THEOREM 3.2. Let $Y$ be a cocycle on $G$ having a trajectory that is $\mathrm{B}_{m}^{2}$ a.p. for some Banach mean $m$ on $L^{\infty}(\mathbb{R})$. Then $Y$ is trivial.

Carlson [2; 7, Theorem 25] has proved that if almost all trajectories of a Borel cocycle are $\mathrm{B}^{2}$ a.p. and have spectra in a fixed countable set, then the cocycle is trivial. For continuous cocycles, Theorem 3.2 generalizes Carlson's result. In fact, the 
afore-mentioned theorem of Agnew and Morse ensures the existence of a Banach mean $m$ on $L^{\infty}(\mathbb{R})$ such that each function in $L^{\infty}(\mathbb{R})$ which is $\mathrm{B}^{2}$ a.p. is also $\mathrm{B}_{m}^{2}$ a.p. Let us note in passing that Theorem 3.1 implies that the spectra (determined either by non-zero Fourier coefficients in the usual sense, if these exist, or by non-zero Fourier coefficients defined with the use of Banach means) of trajectories of a continuous cocycle are contained in a trajectory-independent countable set.

Before proceeding further, we recall some concepts and results from harmonic analysis.

A function $f$ in $L^{\infty}(\mathbb{R})$ is said to be ergodic if its mean value $m(f)$ is independent of the choice of the Banach mean $m$ on $L^{\infty}(\mathbb{R})$ (cf. [11]). The ergodic elements of $L^{\infty}(\mathbb{R})$ can be characterized as those $f \in L^{\infty}(\mathbb{R})$ for which the closed convex envelope of $\left\{T_{s} f: s \in \mathbb{R}\right\}$ contains a constant function. It easily follows from this characterization that for each ergodic function $f$ in $L^{\infty}(\mathbb{R})$, the limit

$$
\lim _{T \rightarrow \infty} \frac{1}{2 T} \int_{x-T}^{x+T} f(t) d t \quad(x \in \mathbb{R})
$$

exists with uniform convergence in $x$, and represents the unique mean value of $f$. A partial converse holds true, as follows. If $f$ is a complex bounded uniformly continuous function on $\mathbb{R}$ for which the above limit exists with uniform convergence in $x$, then $f$ is ergodic (cf. [14]).

A function $f$ in $L^{\infty}(\mathbb{R})$ is said to be totally ergodic if for every $\mu \in \mathbb{R}$, the function $x \rightarrow f(x) e^{i \mu x}$ is ergodic. A complex locally integrable function $f$ is called RyllNardzewski almost periodic (or, more briefly, $R$ a.p.) if, for every $\mu \in \mathbb{R}$, the limit

$$
\lim _{T \rightarrow \infty} \frac{1}{2 T} \int_{x-T}^{x+T} f(t) e^{i \mu t} d t \quad(x \in \mathbb{R})
$$

exists with uniform convergence in $x$ (cf. [9]). Any totally ergodic function in $L^{\infty}(\mathbb{R})$ is $R$ a.p. and its usual Fourier coefficients coincide with the corresponding Fourier coefficients defined with the use of Banach means. Every R a.p. bounded uniformly continuous function on $\mathbb{R}$ is totally ergodic.

A function $f$ in $L^{\infty}(\mathbb{R})$ is called almost totally ergodic if given two Banach means $m$ and $n$ on $L^{\infty}(\mathbb{R})$, the sequences $\left(\mathscr{F}_{m} f(\mu)\right)_{\mu \in \mathbb{R}}$ and $\left(\mathscr{F}_{n} f(\mu)\right)_{\mu \in \mathbb{R}}$ are linearly dependent (cf. [3]).

As an immediate consequence of Theorems 2.3, 2.4, and 3.1, we obtain the following generalization of a result of [3].

THEOREM 3.3. Every trajectory of a cocycle $Y$ on $G$ is almost totally ergodic. If a trajectory of $Y$ is $\mathrm{R}$ a.p., then $Y$ is either non-trivial or c-trivial according as the spectrum of the trajectory is void or not. If, conversely, $Y$ is either non-trivial or c-trivial, then each of its trajectories is $\mathrm{R}$ a.p., and has a non-void spectrum precisely when $Y$ is c-trivial.

We conclude by posing the following problem. Given a trivial cocycle $Y$ on $G$ with a standard representation $(\gamma, X)$ and an element $g$ of $G$, the constants $c(Y, g, m)$ appearing in the statement of Theorem 3.1 form, as $m$ runs over all Banach means on $L^{\infty}(\mathbb{R})$, a closed convex set $C$. The numbers of the form $\phi(X)$, where $\phi$ is a linear multiplicative functional on $L^{\infty}(G)$ localized at $g$, are extreme points of $C$. Are these all the extreme points of $C$ ? 


\title{
References
}

1. R. P. AGNEw and A. P. MoRSE, 'Extensions of linear functionals, with applications to limits, integrals, measures, and densities', Ann. of Math. 39 (1930) 20-30.

2. C. G. R. Carlson, 'Cohomology classes in harmonic analysis', Ph.D. dissertation, Stanford University 1972.

3. W. ChOJNACKI, 'The generalized spectral analysis of $i^{-1}(d / d x)+q$ with real almost periodic $q$, with applications to harmonic analysis', Rend. Mat. (7) 4 (1984) 221-240.

4. K. DE LEEUW, 'On the adjoint semi-groups and some problems in the theory of approximation', Math. Z. 73 (1960) 219-234.

5. R. E. EDWARDS, Functional analysis, theory and applications (Holt, Rinehart \& Winston, New York 1965).

6. H. FURSTENBERG, 'Strict ergodicity and transformations of the torus', Amer. J. Math. 83(1961) 573-601.

7. H. Helson, 'Analycity on compact Abelian groups', Algebras in analysis (ed. J. H. Williamson, Academic Press, London 1975) pp. 1-62.

8. H. HELSON and J.-P. KAHANE, 'Compact groups with ordered duals III', J. London Math. Soc. (2) 4 (1972) 573-575.

9. J.-P. KAHANE, 'Sur les fonctions presque périodiques généralisées dont le spectre est vide', Studia Math. 21 (1962) 231-236.

10. C. KURATOWSKI, Topologie, Vol. I (Państwowe Wydawnictwo Naukowe, Warszawa 1958).

11. Fr. LuST-PiQuaRD, 'Éléments ergodiques et totalement ergodiques dans $L^{\infty}(\Gamma)$ ', Studia Math. 69 (1981) 191-225.

12. J. M. Rosenblatt, 'The number of extensions of an invariant mean', Compositio Math. 33 (1976) 147-159.

13. Z. SemadenI, Banach spaces of continuous functions, Vol. I (Państwowe Wydawnictwo Naukowe, Warszawa 1971).

14. G. S. WooDWARD, 'The generalized almost periodic part of an ergodic function', Studia Math. 50 (1974) 103-116.

\author{
Instytut Matematyki \\ Uniwersytet Warszawski \\ Pałac Kultury i Nauki, IX p. \\ 00-901 Warszawa \\ Poland
}

